\title{
Complete chloroplast genome of Macadamia integrifolia confirms the position of the Gondwanan early-diverging eudicot family Proteaceae
}

\author{
Catherine J Nock*, Abdul Baten, Graham J King \\ From Asia Pacific Bioinformatics Network (APBioNet) Thirteenth International Conference on Bioinformatics \\ (InCoB2014) \\ Sydney, Australia. 31 July - 2 August 2014
}

\begin{abstract}
Background: Sequence data from the chloroplast genome have played a central role in elucidating the evolutionary history of flowering plants, Angiospermae. In the past decade, the number of complete chloroplast genomes has burgeoned, leading to well-supported angiosperm phylogenies. However, some relationships, particulary among early-diverging lineages, remain unresolved. The diverse Southern Hemisphere plant family Proteaceae arose on the ancient supercontinent Gondwana early in angiosperm history and is a model group for adaptive radiation in response to changing climatic conditions. Genomic resources for the family are limited, and until now it is one of the few early-diverging 'basal eudicot' lineages not represented in chloroplast phylogenomic analyses.

Results: The chloroplast genome of the Australian nut crop tree Macadamia integrifolia was assembled de novo from Illumina paired-end sequence reads. Three contigs, corresponding to a collapsed inverted repeat, a large and a small single copy region were identified, and used for genome reconstruction. The complete genome is $159,714 \mathrm{bp}$ in length and was assembled at deep coverage (3.29 million reads; $2000 \mathrm{x}$ ). Phylogenetic analyses based on 83-gene and inverted repeat region alignments, the largest sequence-rich datasets to include the basal eudicot family Proteaceae, provide strong support for a Proteales clade that includes Macadamia, Platanus and Nelumbo. Genome structure and content followed the ancestral angiosperm pattern and were highly conserved in the Proteales, whilst size differences were largely explained by the relative contraction of the single copy regions and expansion of the inverted repeats in Macadamia.
\end{abstract}

Conclusions: The Macadamia chloroplast genome presented here is the first in the Proteaceae, and confirms the placement of this family with the morphologically divergent Plantanaceae (plane tree family) and Nelumbonaceae (sacred lotus family) in the basal eudicot order Proteales. It provides a high-quality reference genome for future evolutionary studies and will be of benefit for taxon-rich phylogenomic analyses aimed at resolving relationships among early-diverging angiosperms, and more broadly across the plant tree of life.

\section{Background}

Chloroplasts are the plastid organelles responsible for photosynthesis, and their genomes have proven to be a valuable resource for plant phylogenetics, population genetics, species identification and genetic engineering. High-throughput next generation sequencing (NGS)

\footnotetext{
* Correspondence: cathy.nock@scu.edu.au

Southern Cross Plant Science, Southern Cross University, Military Road, NSW, Lismore, 2480, Australia
}

technologies have led to a rapid growth in the number of available chloroplast $(\mathrm{cp})$ genomes, including representatives of most major lineages of green plants, Viridiplantae [1]. The quadripartite structure of the plant cp genome is highly conserved, with an inverted repeat region separating the small and large single repeat regions in most species [2].

Molecular phylogenomic studies utilising cp genome sequence data from the genes and slowly-evolving inverted 
repeat regions have been applied to unravel the deep-level evolutionary relationships of plant taxa [1-5], producing robust phylogenies that are corroborated by sequence data from mitochondrial and nuclear genomes [6]. Although cp genome phylogenies have been enormously important in resolving relationships among the flowering plants Angiospermae, the position of some lineages remains unresolved. Relationships among early-diverging lineages, including basal angiosperms, Magnoliidae (magnoliids), Monocotyledoneae (monocots) and basal Eudicotyledoneae (eudicots) have been among the most problematic due to rapid diversification early in the history of flowering plants [7]. Increased taxon sampling, particularly for taxa representing deep-level divergences, may provide resolution. $[8,9]$.

The basal eudicot order Proteales contains the families Nelumbonaceae, Platanaceae and Proteaceae [10]. Fossil evidence and fossil-calibrated molecular dating indicate family-level divergence within the order by the early Cretaceous, over 110 million years ago [11]. There is evidence for long-term morphological and molecular stasis in the Nelumbonaceae and Platanaceae, and the only extant genera Nelumbo and Platanus are both regarded as 'living fossils' [12]. By contrast, the Southern Hemisphere family Proteaceae is morphologically and ecologically diverse. Approximately 79 genera and 1700 species are recognised, including the Australian Banksia and Macadamia and African Protea. Current distribution is the result of both vicariance during Gondwanan breakup and long-distance dispersal [13].

The Proteaceae exhibits remarkably variable levels of endemism and species-richness, notably in the Mediterranean climate biodiversity hotspots of Southwest Australia and the Cape Floristic Region [14,15]. It is, therefore, a family of great interest for studies of speciation, diversification, biogeography and evolution [16-18]. However, genomic resources for the Proteaceae are limited and little is known of the composition and organisation of the genomes and their evolution. Here, as part of an ongoing effort to establish a comprehensive understanding of the macadamia genomes, we present the complete and annotated DNA sequence for the chloroplast from Macadamia integrifolia, to our knowledge the first in the Proteaceae. Given that the closest reference sequences of Platanus and Nelumbo are over 100 million years divergent, the Macadamia cp genome was assembled de novo at deep coverage.

\section{Results}

\section{De novo genome assembly}

After trimming for low quality bases and adapter sequences, there were $1.54 \times 10^{8}$ reads with an average read length of 105 base pairs (bp). De novo assembly produced 540,582 contiguous sequences (contigs) with an N50 of 2,540. The maximum and average contig lengths were 300,523 and 1,032 respectively. Three chloroplast contigs were identified, with greatest similarity to Platanus occidentalis based on total alignment score and percentage sequence identity. These contigs totalled $133,617 \mathrm{bp}$ in length and corresponded to the large single copy $(88,300$ bp), small single copy (18,888 bp) and a double-coverage, collapsed consensus of the inverted repeat regions $(26,429)$. They were aligned to the Platanus cp genome using MUMmer as a starting point to order and assemble the draft genome (Fig. S1 in Additional File 1). The single collapsed inverted repeat (IR) contig was separated into two repeat regions. Assembly of the two IR and the large single copy (LSC) and small single copy (SSC) contigs covered the complete sequence without gaps. Iterations of assembly, realignment and editing using BWA, MUMmer and Gap5 were performed to complete the genome assembly. Sanger sequences spanning the inverted repeat and de novo contig junctions confirmed those in the final assembly. Reference mapping of paired-end reads was used to determine quality and coverage of the finished Macadamia cp genome. Following re-assembly of reads, the 26,429 nucleotide positions of each inverted repeat region were examined for differences and found to be identical. In total, 3.29 million reads $(2.12 \%)$ were mapped. Median coverage was 1,999 times and the minimum coverage of any position was 600 .

\section{Chloroplast genome of Macadamia integrifolia and comparative analyses}

The cp genome of $M$. integrifolia is 159,714 bp in length with a typical quadripartite structure [Genbank: KF862711, Figure 1]. The LSC, SSC and IR regions are $88,093,18,813$ and 26,404 bp respectively and GC content is $38.1 \%$. Gene content and order is identical in Macadamia, Platanus and Nelumbo with each sharing 79 protein-coding, 30 tRNA and 4 rRNA genes. Size differences among Proteales cp genomes are primarily due to expansion of the IR and corresponding reductions in the LSC and SSC regions in Macadamia relative to Platanus and Nelumbo. Indels are located primarily in noncoding regions with the largest a 1,749 bp deletion in Macadamia relative to Platanus in the $n d h C$ to $t r n V-U A C$ intergenic spacer (Table 1, Figure 2). Based on internal stop codons, ycf68 in the Macadamia cp genome is a pseudogene, as in Platanus and Nelumbo and many other angiosperms. In Macadamia ycf15 is intact, with an amino acid sequence identical to many other angiosperms including the magnoliid Calycanthus floridus. In Platanus, ycf15 is a pseudogene [19], likely due to a 597 bp deletion in the $y c f 15$ coding region relative to Macadamia (Figure 2). The rps19 gene is located at the 3'-end of the IR regions in Macadamia and Nelumbo, and spans the $\mathrm{IR}_{\mathrm{A}}$-LSC and LSC-IR $\mathrm{B}$ junction in Platanus only. The presence of ACG start codons in $n d h D$, psbL and $r p l 2$ suggests that RNA editing is required for translation of these genes in Macadamia and Platanus. 


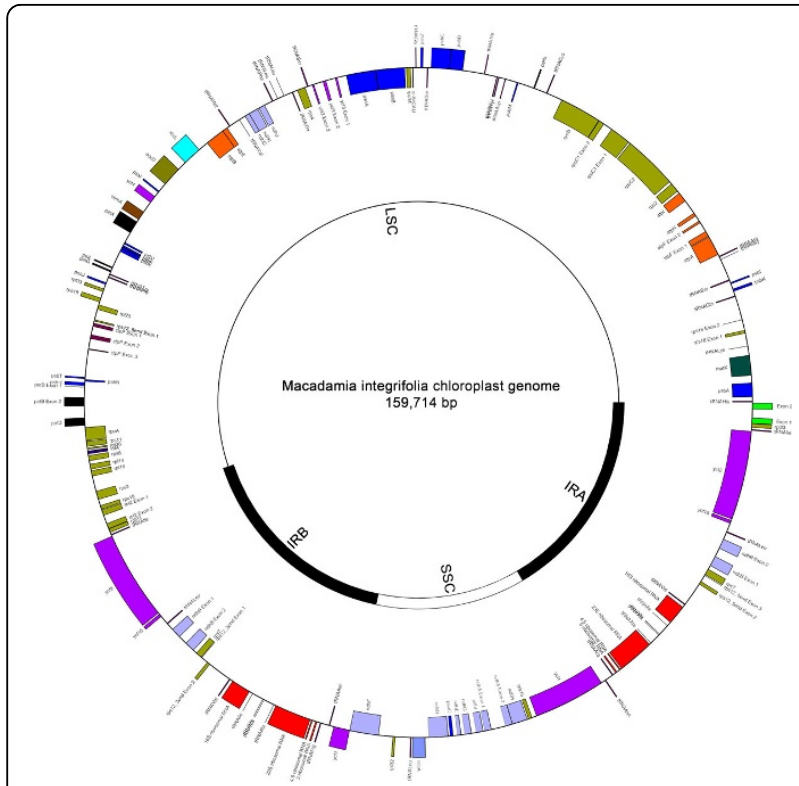

Figure 1 Chloroplast genome map of Macadamia integrifolia Organisation of the chloroplast genome of Macadamia integrifolia (Proteaceae) showing annotated genes. The inner circle shows the location of the large and small single copy (LSC and SSC) and inverted repeat regions $\left(\mathrm{R}_{\mathrm{A}}\right.$ and $\left.I \mathrm{R}_{B}\right)$

\section{Characterisation of cpSSR loci}

In total, 59 chloroplast simple sequence repeat (cpSSR) regions were identified in Macadamia. Of these, 57 were mononucleotide (A/T) and two were dinucleotide (AT/TA) repeats. The majority (79\%), were located in noncoding sections of the LSC region. However, $14 \mathrm{cpSSR}$ are located in exons including two in $y c f 1$ replicated in the inverted repeat regions. No tri- or tetranucleotide repeats over $15 \mathrm{bp}$ in length were found. Of particular interest for population genetics studies are regions of the $c l p P$ intron (660bp) and trnK to rps16 intergenic spacer (818bp) containing multiple SSRs as they are co-located in short sections amenable to PCR amplification and Sanger sequencing. The 13 cpSSRs in noncoding regions shared with Platanus are also of interest as they are likely to be present and may be variable in other Proteaceae species (Table 2).

\section{Phylogenetic analyses}

Maximum likelihood (ML) analyses were performed on 87-taxa chloroplast gene and 160-taxa IR alignments in order to determine the position of Macadamia within Angiospermae, and the consequence of its inclusion on inferring phylogenetic relationships among basal eudicots.

\section{Chloroplast Gene Phylogeny}

The final 87-taxa and 83-gene alignment used for analyses was $66,738 \mathrm{bp}$ in length. The proportion of gaps and undetermined characters was $4.04 \%$, and GC content was $38.4 \%$. The optimal partitioning scheme identified under the Bayesian information criteria (BIC) using relaxed clustering analysis in PartitionFinder $(\ln L=-1081518.0$; BIC 2170800.8) contained 49 partitions. Maximum likelihood analyses under the 49-partition and single partition (hereafter unpartitioned) strategies and the GTR $+\Gamma$ model produced identical topologies. The ML 'best' tree with the highest likelihood score $(\ln L=-1087999.5)$ produced by the partitioned ML analysis (Figure 3; Fig. S2 in Additional File 2) shared the same topology as the best tree from unpartitioned analysis $(\operatorname{lnL}=-1110496.6)$.

\section{Inverted repeat region phylogeny}

The final IR alignment used for analyses was 24,693 bp in length, including $10,781 \mathrm{bp}(43.7 \%)$ of non-coding sequence from spacers and introns. The proportion of gaps and undetermined characters was $13.2 \%$ and GC content was $42.5 \%$. The optimal partitioning scheme in PartitionFinder ( $\ln \mathrm{L}=-140178.1$; BIC 296837.1) contained 5 partitions. Maximum likelihood analyses under the 5-partition and unpartitioned strategies with the $\mathrm{GTR}+\Gamma$ model produced identical topologies. The $\mathrm{ML}$ 'best' tree $(\ln L=-261860.8)$ produced by the partitioned analysis (Figure 4; Fig. S3 in Additional File 3) shared the same topology as the best tree from unpartitioned analysis $(\operatorname{lnL}=-288975.8)$.

Phylogenetic analyses based on both chloroplast genes and inverted repeat regions provided maximum bootstrap (BS) support for a sister relationship between Macadamia and Platanus, and for a Proteales clade also containing Nelumbo (BS 100\%). Sabiaceae (Meliosma) was sister to the Proteales in all analyses, however, the level of support for this clade was lower in the IR (BS 53\%) compared to the 83-gene (BS 70\%) partitioned analyses (Figure 3, Figure 4). The 83-gene and IR phylogenies were highly congruent, with the only differences among basal eudicot taxa in the position of Buxus and Trochodendron. In the 83-gene phylogeny, support for a Buxus versus Trochondendron sister relationship to the core eudicots was marginal (BS 50\%), whereas Trochodendron was sister to the core eudicots (= Gunneridae) in the partitioned (BS 90\%) and unpartitioned (BS 86\%) IR analyses respectively. The main topological difference among core eudicots was in the position of the three major clades: superrosids, superasterids and Dilleniaceae. In cp-gene phylogenies, Dillenia was sister to the superrosids (BS 90\%) and in IR phylogenies Dillenaceae was sister to the superosids+superasterids (BS 80\%).

\section{Discussion}

\section{Characteristics of the Macadamia cp genome and comparison to other angiosperms}

The chloroplast genome of Macadamia integrifolia cultivar HAES 741 was sequenced at deep coverage ( 2000x) and assembled de novo using Illumina NGS reads. Structure, gene content and order appear to be highly conserved in the basal eudicot order Proteales, and in comparision to the inferred ancestral cp genome 
Table 1 Characteristics of Proteales chloroplast genomes and primary noncoding indels contributing to length differences, in base pairs

\begin{tabular}{lccc}
\hline Region & Macadamia & Platanus & Nelumbo \\
\hline Chloroplast genome & 159714 & 161791 & 163307 \\
Inverted Repeats, IR And IR & 26404 & 25066 & 26065 \\
ycf2 to trnL-CAA spacer & 1014 & 424 & 1022 \\
trnl-GAU intron & 946 & 942 & 760 \\
Small Single Copy, SSC & 18813 & 19509 & 19330 \\
ndhG to ndhl spacer & 362 & 380 & 467 \\
ndhA intron & 2194 & 2187 & 1915 \\
rpl32 to trnL-UAG spacer & 1149 & 647 & 1070 \\
Large Single Copy, LSC & 88093 & 92150 & 91847 \\
rpsi6 to trnQ-UUG spacer & 1784 & 1299 & 2051 \\
ndhC to trnV-UAC spacer & 517 & 2266 & 2156 \\
ycf3 to trnS-GGA spacer & 308 & 889 & 926 \\
petA to psbJ spacer & 841 & 984 & 1105 \\
trnT-UGU to trnL-UAA spacer & 649 & 1419 & 1031 \\
IR -SSC Junction & & & 1668 \\
trnN-GUU to ndhF spacer & 1555 & 746 & 5520 \\
SSC-IRA Junction & & 5748 & \\
ycf 1 & 5538 & & \\
\hline
\end{tabular}

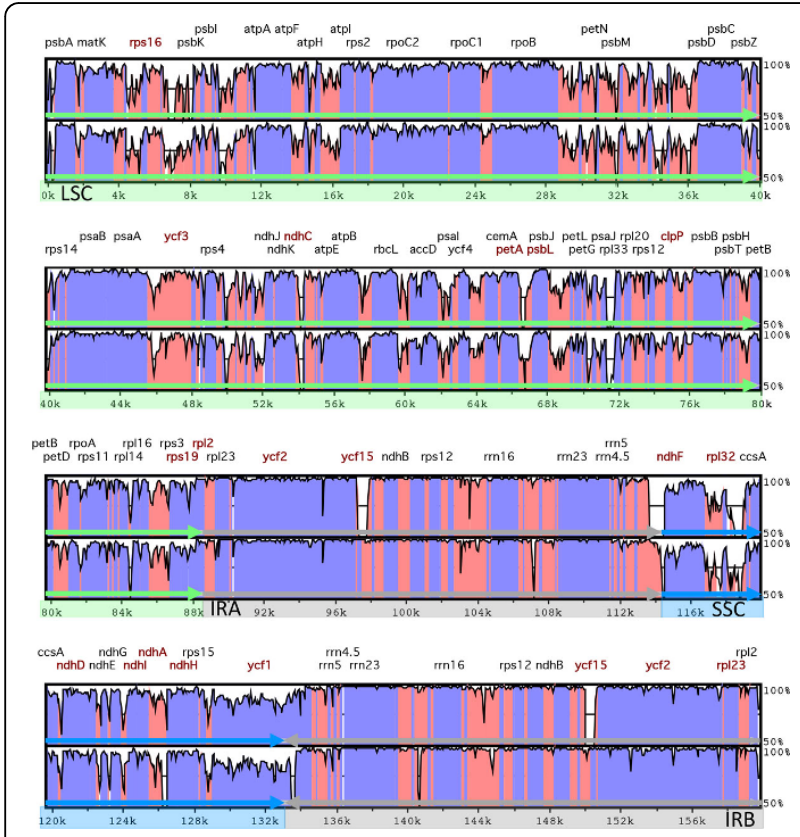

Figure 2 Sequence identity plot comparing the chloroplast genome of Macadamia to other Proteales. Pairwise comparisons between Macadamia integrifolia and chloroplast genomes from the Proteales genera Platanus (top) and Nelumbo (bottom) using mVISTA. The $\boldsymbol{y}$-axis represents \% identity ranging from $50-100 \%$. Coding and non-coding regions are marked in purple and pink respectively. Also shown are the genes discussed in the text (red), large single copy (LSC, green), small single copy (SSC, blue) and inverted repeat (IR, grey) regions. organization of Nicotiana tabacum and many other angiosperms [20]. Consistently high levels of conservation within Angiospermae are indicative of evolutionary constraints on the cp genome of photosynthetic plants [21]. Major differences among angiosperm cp genomes are due to gene losses, inversions and expansion/contraction of inverted repeat regions. Gene loss in parasitic plants can lead to markedly reduced cp genome size. For example, the cp genome of the underground orchid Rhizanthella gardneri is only $59 \mathrm{~kb}$ [22] and there may have been a complete loss of the plastid genome in Rafflesia lagascae [23]. Gene loss can also be due to the transfer of cp genes to the nuclear genome [24]. Gene order is largely conserved among angiosperms including Macadamia and other basal eudicots, however, large inversions altering gene order have been reported in some core eudicot species [e.g. 25,26]. The main effects of expansion and contraction of the IR regions at the LSC and SSC junctions are the formation of pseudogenes, and changes in genome size and evolutionary rate [24]. The smaller cp genome of Macadamia compared to Platanus and Nelumbo is primarily due to relative reduction of the single copy regions, with most deletions in intergenic spacers and introns. The Macadamia IR at $26.4 \mathrm{~kb}$ is the largest yet reported in the Proteales but is considerably smaller than those of the basal eudicot Trochondendron (30.7 kb) and the core eudicot Pelargonium x hortorum, (76 kb) [25,27]. Proteales cp genomes also differ in the complement of pseudogenes. 
Table 2 Distribution of Macadamia integrifolia chloroplast simple sequence repeat (cpSSR) regions

\begin{tabular}{|c|c|c|c|c|c|c|c|c|c|c|c|c|c|}
\hline \multirow{2}{*}{$\frac{\text { cpSSR }}{1}$} & \multirow{2}{*}{$\frac{\text { repeat motif }}{A}$} & \multirow{2}{*}{$\frac{\text { Length bp }}{15}$} & \multirow{2}{*}{$\begin{array}{l}\text { start } \\
273\end{array}$} & \multirow{2}{*}{$\begin{array}{c}\text { end } \\
288\end{array}$} & \multicolumn{2}{|c|}{ Region } & \multirow{2}{*}{$\begin{array}{l}\text { cpSSR } \\
32\end{array}$} & \multirow{2}{*}{$\frac{\text { repeat motif }}{A}$} & \multirow{2}{*}{$\frac{\text { Length bp }}{10}$} & \multirow{2}{*}{$\frac{\text { start }}{67617}$} & \multirow{2}{*}{$\begin{array}{l}\text { end } \\
67627\end{array}$} & \multicolumn{2}{|c|}{ Region } \\
\hline & & & & & LSC & $\operatorname{trnH}-p s b A$ & & & & & & LSC & psbF exon \\
\hline 2 & A & 10 & 1845 & 1855 & LSC & trnK-matK & 33 & A & 13 & 68887 & 68900 & LSC & psbE-petL \\
\hline 3 & A & 13 & 4462 & 4475 & LSC & trnK-rps 16 & 34 & $\mathrm{~T}$ & 12 & 70983 & 70995 & LSC & psaJ-rp/33 \\
\hline $4^{b}$ & $\mathrm{~T}$ & 11 & 5269 & 5280 & LSC & trnK-rps16 & 35 & A & 10 & 72914 & 72924 & LSC & rpl20-rps 12 \\
\hline 5 & $\mathrm{~T}$ & 15 & 6997 & 7012 & LSC & rps16-trnQ & 36 & A & 16 & 72943 & 72959 & LSC & rpl20-rps 12 \\
\hline 6 & A & 10 & 7141 & 7151 & LSC & rps16-trnQ & $37^{a, b}$ & $\mathrm{~T}$ & 10 & 74379 & 74389 & LSC & clpP intron \\
\hline 7 & A & 12 & 9935 & 9947 & LSC & trns-trnG & 38 & $\mathrm{~T}$ & 10 & 74633 & 74643 & LSC & clpP intron \\
\hline $8^{b}$ & $\mathrm{~T}$ & 14 & 11345 & 11359 & LSC & $\operatorname{trn} G-\operatorname{trn} R$ & 39 & A & 11 & 75028 & 75039 & LSC & clpP intron \\
\hline 9 & A & 17 & 11514 & 11531 & LSC & $\operatorname{trnR}$-atpA & $40^{a}$ & A & 14 & 81691 & 81705 & LSC & petD-rpoA \\
\hline $10^{a}$ & A & 10 & 13186 & 13196 & LSC & atpA-atpF & $41^{a}$ & $\mathrm{~T}$ & 13 & 83842 & 83855 & LSC & infa-rps 8 \\
\hline $11^{b}$ & A & 11 & 14349 & 14360 & LSC & atpF intron & 42 & $\mathrm{~T}$ & 17 & 84941 & 84958 & LSC & rp/14-rp/16 \\
\hline 12 & $\mathrm{~T}$ & 11 & 15497 & 15508 & LSC & atpH-atpl & 43 & $\mathrm{~T}$ & 12 & 86421 & 86433 & LSC & rps16-rps3 \\
\hline 13 & $\mathrm{~T}$ & 10 & 17839 & 17849 & LSC & rps2 exon & 44 & AT & 16 & 4782 & 4798 & LSC & trnK-rps 16 \\
\hline 14 & A & 10 & 18105 & 18115 & LSC & rps2-rpoC2 & 45 & AT & 16 & 36068 & 36084 & LSC & $\operatorname{trnT} T-p s b D$ \\
\hline $15^{b}$ & $\mathrm{~T}$ & 10 & 20185 & 20195 & LSC & rpoc2 exon & & & & & & & \\
\hline $16^{a, b}$ & $\mathrm{~T}$ & 11 & 20316 & 20327 & LSC & rpoc2 exon & 46 & $\mathrm{~T}$ & 11 & 88100 & 88111 & $\mathbb{I}_{B}$ & rps19-rp/2 \\
\hline 17 & $\mathrm{~T}$ & 12 & 22934 & 22946 & LSC & rpoc1 exon & 47 & $\mathrm{~T}$ & 10 & 114378 & 114388 & $\mathbb{R}_{B}$ & ycfl exon \\
\hline 18 & A & 11 & 30325 & 30336 & LSC & $\operatorname{trnC}$-petN & 48 & $\mathrm{~T}$ & 10 & 114477 & 114487 & $\mathbb{R}_{B}$ & ycfi exon \\
\hline 19 & $\mathrm{~T}$ & 10 & 32312 & 32322 & LSC & psbM-trnD & & & & & & & \\
\hline $20^{a}$ & $\mathrm{~T}$ & 10 & 34365 & 34375 & LSC & $\operatorname{trn} E-\operatorname{trn} T$ & $49^{b}$ & $\mathrm{~T}$ & 18 & 117406 & 117424 & SSC & ndhF-rp/32 \\
\hline 21 & A & 12 & 35944 & 35956 & LSC & $\operatorname{trnT} T-p s b D$ & 50 & A & 10 & 125592 & 125602 & SSC & $n d h A$ intron \\
\hline $22^{a}$ & $\mathrm{~T}$ & 10 & 39082 & 39092 & LSC & $p s b c-t r n s$ & 51 & A & 14 & 125805 & 125819 & SSC & $n d h A$ intron \\
\hline 23 & A & 16 & 39929 & 39945 & LSC & psbZ-trnG & 52 & $\mathrm{~T}$ & 10 & 128234 & 128244 & SSC & ndhH-rps 15 \\
\hline $24^{a}$ & A & 15 & 48007 & 48022 & LSC & $y c f 3$ intron & 53 & $\mathrm{~T}$ & 12 & 129915 & 129927 & SSC & ycfl exon \\
\hline 25 & A & 12 & 48271 & 48283 & LSC & $y c f 3-t r n s$ & 54 & A & 15 & 130143 & 130158 & SSC & ycfl exon \\
\hline 26 & $\mathrm{~T}$ & 14 & 49898 & 49912 & LSC & $\operatorname{trn} T-\operatorname{trn} L$ & $55^{a}$ & A & 11 & 132293 & 132304 & SSC & ycfl exon \\
\hline 27 & $\mathrm{~T}$ & 14 & 51057 & 51071 & LSC & $\operatorname{trnL}-t r n F$ & 56 & $\mathrm{~T}$ & 10 & 132660 & 132670 & SSC & ycfl exon \\
\hline 28 & $\mathrm{~T}$ & 13 & 51972 & 51985 & LSC & $\operatorname{trnF-ndhJ}$ & & & & & & & \\
\hline 29 & $\mathrm{~T}$ & 10 & 52600 & 52610 & LSC & $n d h J-n d h K$ & 57 & A & 10 & 133320 & 133330 & $\mathbb{I R}_{\mathrm{A}}$ & ycfl exon \\
\hline $30^{a, b}$ & $\mathrm{~T}$ & 10 & 55335 & 55345 & LSC & trnM-atpE & 58 & A & 10 & 133419 & 133429 & $\mathbb{I} R_{A}$ & ycfl exon \\
\hline 31 & $\mathrm{~T}$ & 14 & 63629 & 63643 & LSC & ycf4-cemA & 59 & A & 11 & 159696 & 159707 & $\mathbb{I R}_{\mathrm{A}}$ & rpl2-trnH \\
\hline cpSSR r & gions also pr & in Platc & us (a), Ne & elumbo & & & & & & & & & \\
\hline
\end{tabular}

The intact ycf15 gene of Macadamia is, in Platanus a pseudogene due to a large deletion. The function and validity of $y c f 15$ are uncertain, and there is no evidence of chloroplast-nuclear gene transfer in angiosperms with intact or disabled $y c f 15$ genes [28]. The rps19 gene spans the $\mathrm{IR}_{\mathrm{A}}$-LSC junction causing an pseudogene in the $\mathrm{IR}_{\mathrm{B}}$ of Platanus.

Phylogenetic implications and the position of Proteaceae Australia is the origin and centre of diversity of the Proteaceae, and this morphologically distinct and diverse family is distributed across remnant landmasses of the southern supercontinent Gondwana [15]. The order Proteales inclusive of Proteaceae, Platanaceae and Nelumbonaceae was established relatively recently, on the basis of molecular data, and morphological synapomorphies for the order are yet to be identified [29-31].
The $83 \mathrm{cp}$ gene and IR region alignments used in this study are the largest sequence-rich datasets to include the basal eudicot family Proteaceae. Maximum likelihood phylogenies confirmed the position of Macadamia within the Proteales, and were congruent and largely concordant with recent phlyogenomic studies [1,3-6]. There was maximum support for a sister relationship between Proteaceae and Platanaceae, and for a Proteales clade containing these families and Nelumbonaceae. A 640-taxa angiosperm phylogeny using 17 genes from all three plant genomes included the Proteaceae taxa Petrophile and Roupala [6], and a 57-taxa, $17 \mathrm{~kb}$ alignment of chloroplast introns, spacers and genes included Embothrium and Grevillea [32]. Both studies confirmed inclusion of Proteaceae in the Proteales (BS 100\%), in accordance with the Angiosperm Phylogeny Group III system [10]. In this study, a clade containing Sabiaceae (Meliosma) and Proteales was 


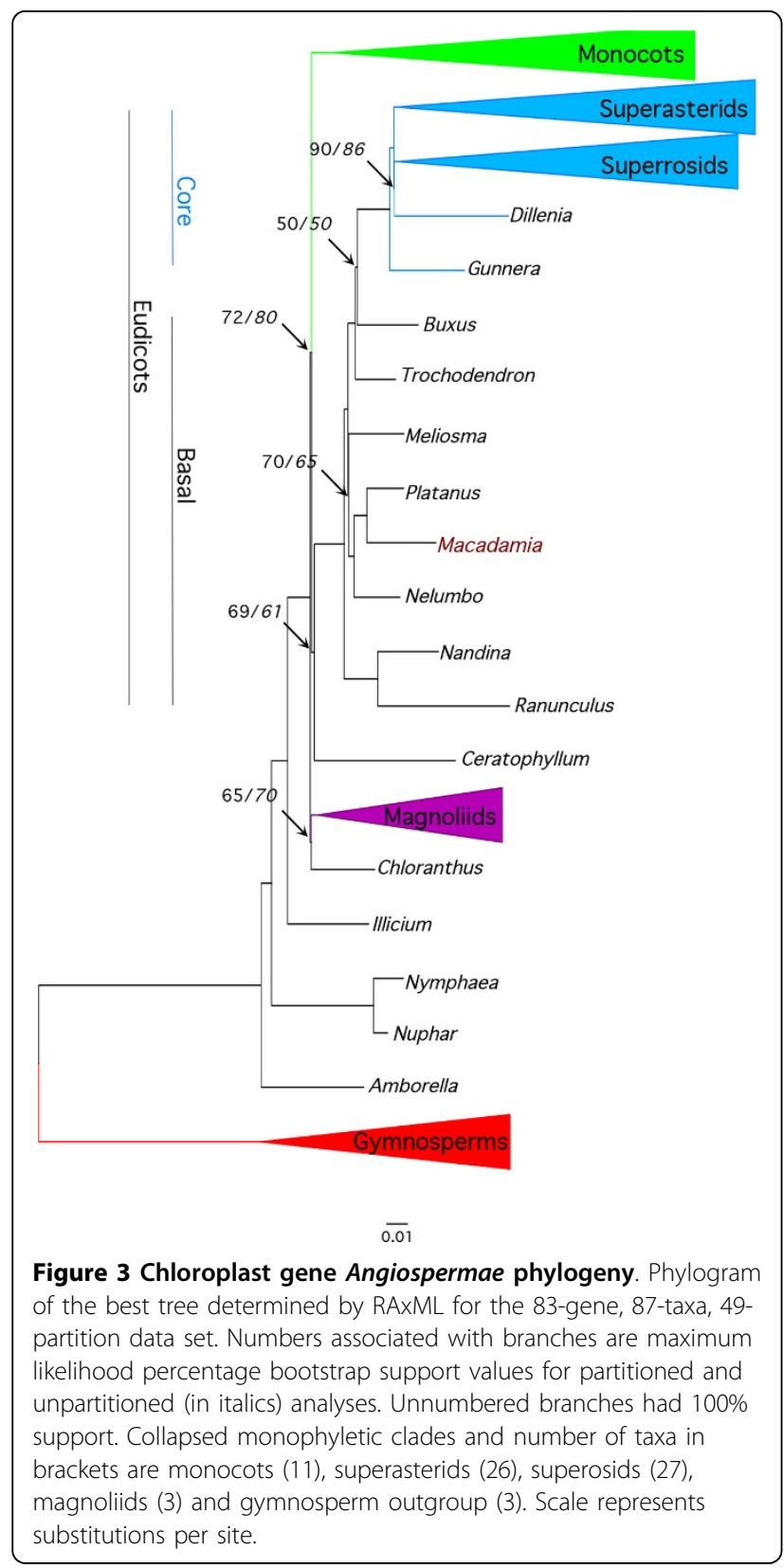

recovered from both the 83-gene and IR analyses with moderate support (Figure 3, Figure 4). These results are consistent with those from previous phylogenomic studies with support values ranging from 43-88\%, although a Proteales-Sabiaceae clade was not recovered in all analyses [1-6,32].

The inclusion of Macadamia in taxon-rich chloroplast gene and inverted repeat alignments produced largely congruent and well-supported ML phylogenies. The main topological differences were in the positions of taxa representing lineages that are unplaced in the APG III system including Dillenia, Trochodendron and Buxus [10]. Within core eudicots, there was conflicting strong support for a

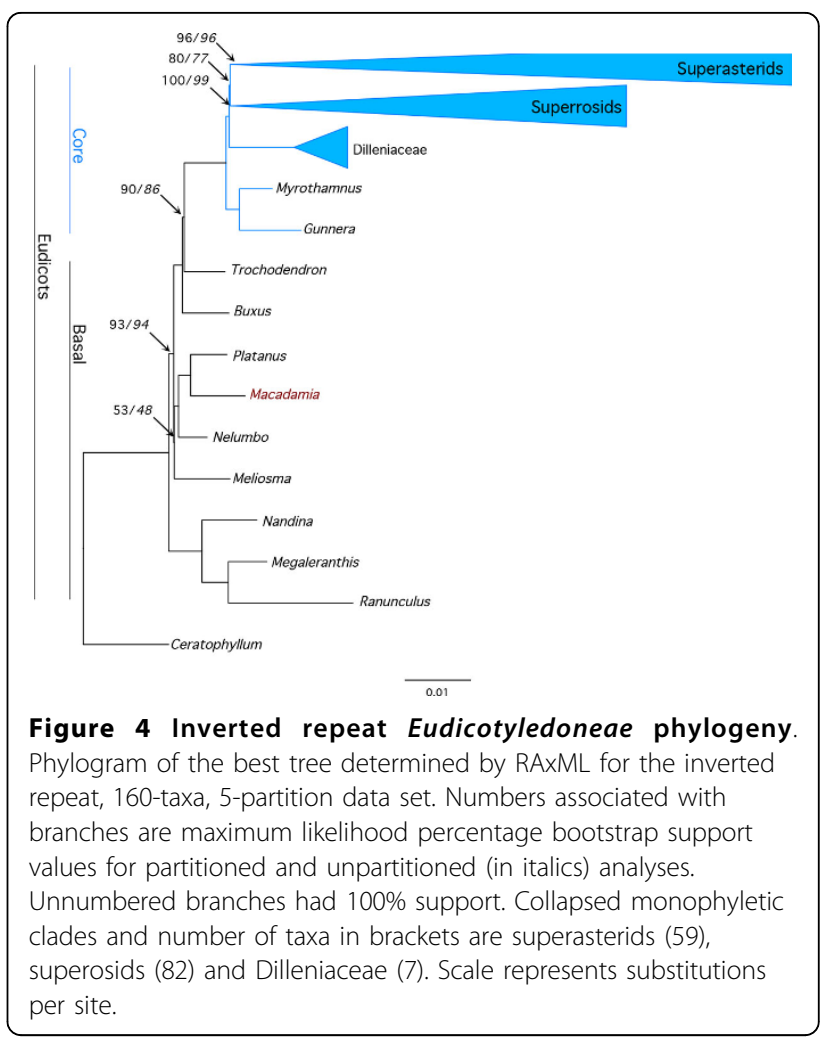

sister relationship between (1) Dillenia and superrosids, and (2) Dillenaceae, represented by 7 genera, and superosids+superasterids, in cp-gene and IR analyses respectively. There was strong support for Trochodendron as sister to core eudicots in IR analyses, whilst the core eudicot sister was undetermined between Trochodendron and Buxus in cp-gene analyses (BS 50\%). Interestingly, previous studies provided strong support for a Buxaceae-core eudicot clade based on data from the $\mathrm{cp}$, mitochondrial and nuclear genes [6] and for an alternative Trochodendron-core eudicot clade using the cp IR region. Efforts to resolve relationships among unplaced angiosperm lineages, are hampered by short internal branch lengths due to rapid divergence of major lineages in the Cretaceous [7]. Full resolution of relationships among basal eudicots may require denser sampling of both taxa and genes.

\section{Utility of the Macadamia chloroplast genome}

Problems in identfying a single locus DNA barcode for plants, and advances in sequencing technologies have led to suggestions that the cp genome could have utility in species identification [33,34]. Possible obstacles include the cost and complexity of assembly [35]. However, the advantages of using a NGS approach to chloroplast DNA barcoding include the potential to eliminate PCR and hence reliance on 'universal' primers. Given the widely reported transfer of chloroplast sequence to 
the nuclear genome [36,37] avoidance of PCR further eliminates the risk of amplifying paralogous nuclear plastid-like sequences (NUPTs). The availability of high quality cp genomes for representatives of each of the 413 recognised angiosperm families should facilitate species identification. This can be achieved through rapid identification of cp sequences by reference mapping of low coverage NGS reads at multiple locations, without the requirement for complete genome assembly. Continual improvements in sequencing technologies, including increased read lengths and decreasing cost, in addition to new methods to optimise recovery of chloroplast sequences from plant DNA $[38,39]$ are bringing cp genome-wide barcoding closer to reality.

Whole chloroplast genome sequencing enables identification of intraspecific variation for phylogeographic studies, even in genetically depauperate species [40] and cPSSR regions have been widely used in population genetics $[41,42]$. The 59 Macadamia cpSSR identified in this study may provide markers with broad utility across Proteaceae species. The Macadamia cp genome is currently providing a reference sequence for inferring the domestication and evolutionary histories of Macadamia (unpublished results). Furthermore, it will be of benefit for taxonrich phylogenomic studies and understanding of the evolution and adaptations underlying the remarkable diversity of this large Southern Hemisphere plant family [31].

\section{Conclusions}

The complete chloroplast genome of Macadamia integrifolia was assembled de novo from Illumina NGS reads, and provides the first reference genome sequence for the Gondwanan plant family, Proteaceae. Despite sequencing at deep coverage ( 2000x) the genome was recovered in three contigs, one of which corresponded to a collapsed copy of the inverted repeat regions. Although genome assembly from these contigs was straightforward, this provides an illustration of the problems that large repeat regions present to de novo genome assembly from NGS short read sequence data. Phylogenetic analyses of both 83-gene and inverted repeat region alignments confirmed the position of Proteaceae in the order Proteales, with maximum support for a sister relationship between Platanaceae (Platanus) and Proteaceae (Macadamia). The Macadamia chloroplast genome provides a high-quality reference for future evolutionary studies within the Proteaceae and will be of benefit for taxon-rich phylogenomic analyses aiming to resolve relationships among early-diverging angiosperms and more broadly across the plant tree of life.

\section{Methods}

\section{Sample Collection}

Fresh leaf material was collected from a single Macadamia integrifolia, cultivar 741 'Mauka' individual from the
Macadamia Varietal Trial plantation M2 at Clunes, New South Wales and stored at $-80^{\circ} \mathrm{C}$ prior to DNA extraction. A voucher specimen was deposited in the Southern Cross University herbarium [accession PHARM-130813].

\section{DNA extraction, library preparation and Illumina sequencing}

Leaf tissue was frozen in liquid nitrogen and ground using a tissue lyser (MM200, Retsch, Haan, Germany). Total genomic DNA was extracted using a DNeasy Plant Maxi kit (Qiagen Inc., Valencia, CA, USA) and quantified using a Qubit dsDNA BR assay (Life Technologies, Carlsbad, CA, USA). Genomic DNA was sheared using a Covaris S220 focused-ultrasonication device (Covaris Inc., Woburn USA) to a mean fragment size of $500 \mathrm{bp}$. A DNA library was prepared using Illumina TruSeq DNA Sample Preparation kit v2 following manufacturer's instructions (Illumina, San Diego, USA). Fragment size distribution and concentration were determined using a DNA 1000 chip on a Bioanalyser 2100 instrument (Agilent Technologies, Santa Clara, USA). Approximately 4 pmol of the library was paired-end sequenced $(150 \times 2$ cycles $)$ on an Illumina GA IIx instrument. Base calling was performed with Illumina Pipeline version 1.7.

\section{De novo assembly of chloroplast genome}

Paired-end sequence reads were trimmed to remove low quality bases $(\mathrm{Q}<20,0.01$ probabilility error) and adapter sequences in CLC Genomics Workbench, version 4.9 (CLC Bio, Aarhus, Denmark; www.clcbio.com). CLC de novo assembler, which utilises de Bruijn graphs for the assembly, was used for the assembly with the option to map reads back to contigs and the following optimised parameters: $\mathrm{k}$-mer $=35$; bubble size $=50$; indel cost $=3$; length fraction $=0.5$; similarity index $=0.8$. In order to identify contigs of $\mathrm{cp}$ origin, assembled sequences were aligned to a local database containing angiosperm complete cp genome sequences from NCBI using BLASTN [43]. Contigs with significant alignment were selected for further analysis. Alignments were visualised using mummer dotplots [44] to estimate the proportion of the genome covered and to order and connect contigs. Quality trimmed reads were mapped back to contigs using Burrows-Wheeler Aligner [45] and contigs were extended and joined using Gap5 [46]. Coverage and quality of the draft genome sequence were assessed by reference mapping of trimmed paired-end reads using CLC Genomics Workbench. To confirm accuracy, sequences spanning contig and repeat region junction regions were PCR amplified using custom primers and Sanger sequenced. The finished genome was annotated using DOGMA (Dual Organellar Genome Annotator) [47] and deposited in Genbank [Genbank:KF862711]. 


\section{Phylogenetic and Comparative Analyses}

To compare structure and gene content within the order Proteales, and to identify variable regions the annotated cp genomes of Macadamia integrifolia, Platanus occidentalis [DQ923116] and Nelumbo nucifera [FJ754270] were aligned using MAFFT version 7.017 [48]. A visual representation of the alignment and regions of interspecific variation was generated in mVISTA [49]. Chloroplast SSR regions were identified using Msatcommander version 1.8.2 specifying minimum lengths of 10,16 , and $24 \mathrm{bp}$ for mono-, di-, and tri- and tetranucleotides respectively [50].

To examine the position of Proteaceae within Angiospermae, genes were extracted from the Macadamia cp genome and added to the 83-gene, 86-taxa alignment of Moore et al. [4] in Geneious Pro, version 7.1.5 (Biomatters Ltd., Auckland, New Zealand). The gymnosperm outgroup taxa were Pinus, Cycas and Ginkgo. To further examine relationships among basal eudicot taxa, the slowly-evolving IR region of the Macadamia cp genome was aligned to a 159-taxa eudicot subset of the inverted repeat alignment of Moore et al. [5] in Geneious Pro with the outgroup taxon Ceratophyllum. Regions of alignment ambiguous sequence data in both matrices, and insertions present in one or few taxa were excluded. For each alignment, PartitionFinder version 1.1.1 was used to select the best-fit partitioning scheme under the Bayesian information criterion with the relaxed clustering algorithm, default 10\% [51]. Data blocks analysed in the 83-gene alignment included first, second and third codon positions for 79 protein-coding genes, and 4 ribosomal RNA genes. Data blocks analysed in the IR alignment included codon positions for 7 protein-coding genes, and 4 ribosomal RNA genes, 7 transfer RNA genes, intergenic spacers and introns. Maximum likelihood analyses on partitioned and unpartitioned datasets were conducted using Randomised Accelerated Maximum Likelihood, RaxML version 7.4.2 with 100 bootstrap replicates and 10 subsequent thorough ML searches under the general time reversable (GTR) substition model and the gamma $(\Gamma)$ model of among site rate heterogeneity [52]. Bootstrap proportions were drawn on the tree with highest likelihood score from the 10 independent searches. Trees were visualised in FigTree version1.4.0.

\section{Additional material}

Additional File 1: Figure S1: Dot plot analysis of Macadamia chloroplast contigs. Dotplot showing identify of three Macadamia de novo assembled chloroplast contigs in comparison to the chloroplast genome of Platanus occidentalis.

Additional File 2: Figure S2: Phylogram of the best ML tree determined by RaxML (InL = -1087999.5) for the 83-gene, 87-taxa and 49-partition data set. Numbers associated with branches are ML percentage bootstrap support values.
Additional File 3: Figure S3: Phylogram of the best ML tree determined by RAxML (InL $=-261860.8$ ) for the inverted repeat region, 160-taxa and 5-partition data set. Numbers associated with branches are $\mathrm{ML}$ percentage bootstrap support values.

\section{Competing interests}

The authors declare that they have no competing interests.

\section{Authors' contributions}

$\mathrm{CN}$ and GK conceived and designed the study. CN performed de novo assembly, genome annotation, phylogenetic and other analyses and drafted the manuscript. $A B$ participated in bioinformatics, genome assembly and phylogenetic analyses. All authors read and approved the final manuscript.

\section{Acknowledgements}

The authors thank Sarah Williams, Roxane Legaie and Dominique Gorse from the Queensland Facility for Advanced Bioinformatics (QFAB) for bioinformatics support. We are grateful to Asuka Kawamata, Mark Edwards, Stirling Bowen and Martin Elphinstone for technical support; and to Michael Moore for providing alignment data [4]. This work was completed as part of the Macadamia genome project, with support from Southern Cross University Division of Research.

\section{Declarations}

The publication costs for this article were funded by Southern Cross University, Lismore, Australia.

This article has been published as part of BMC Genomics Volume 15 Supplement 9, 2014: Thirteenth International Conference on Bioinformatics (InCoB2014): Computational Biology. The full contents of the supplement are available online at http://www.biomedcentral.com/bmcgenomics/ supplements/15/S9.

Published: 8 December 2014

\section{References}

1. Ruhfel BR, Gitzendanner MA, Soltis PS, Soltis DE, Burleigh JG: From algae to angiosperms - inferring the phylogeny of green plants (Viridiplantae) from 360 plastid genomes. BMC Evol Biol 2014, 14:23.

2. Palmer JD, Stein DB: Conservation of chloroplast genome structure among vascular plants. Curr Genet 1986, 10:823-833.

3. Jansen RK, Cai Z, Raubeson LA, Daniell H, dePamphilis CW, Leebens-Mack J, Müller KF, Guisinger-Bellian M, Haberle RC, Hansen AK, Chumley TW, Lee S, Peery R, McNeal JR, Kuehl JV, Boore JL: Analysis of 81 genes from 64 plastid genomes resolves relationships in angiosperms and identifies genome-scale evolutionary patterns. Proc Natl Acad Sci USA 2007, 104:19369-19374.

4. Moore MJ, Soltis PS, Bell CD, Burleigh JG, Soltis DE: Phylogenetic analysis of 83 plastid genes further resolves the early diversification of eudicots. Proc Natl Acad Sci USA 2010, 107(10):4623-4628.

5. Moore MJ, Hassan N, Gitzendanner MA, Bruenn RA, Croley M, Vandeventer A, Horn JW, Dhingra A, Brockington SF, Latvis M, Ramdial J, Alexandre R, Peidrahita A, Xi Z, Davis CC, Soltis PS, Soltis DE: Phylogenetic analysis of the plastid inverted repeat for 244 species: insights into deeper-level angiosperm relationships from a long, slowly evolving sequence region. Int J Plant Sci 2011, 172(4):541-558.

6. Soltis DE, Smith SA, Cellinese N, Wurdack KJ, Tank DC, Brockington SF, Refulio-Rodriguez NF, Walker JB, Moore MJ, Carlsward BS, Bell CD, Latvis M, Crawley S, Black C, Diouf D, Xi Z, Rushworth CA, Gitzendanner MA, Sytsma KJ, Qiu Y, Hilu KW, Davis CC, Sanderson MJ, Beaman RS, Olmstead RG, Judd WS, Donoghue MJ, Soltis PS: Angiosperm phylogeny: 17 genes, 640 taxa. Am J Bot 2011, 98(4):704-730.

7. Bell CD, Soltis DE, Soltis PS: The age and diversification of the angiosperms revisited. Am J Bot 2010, 97:1296-1303.

8. Hillis DM, Pollock DD, McGuire JA, Zwickl DJ: Is sparse taxon sampling a problem for phylogenetic inference? . Syst Biol 2003, 52:124-126.

9. Leebens-Mack J, Raubeson LA, Cui L, Kuehl JV, Fourcade MH, Chumley TW, Boore $J$, Jansen RK, dePamphilis CW: Identifying the basal angiosperm 
node in chloroplast genome phylogenies: sampling one's way out of the Felsenstein Zone. Mol Biol Evol 2005, 22(10):1948-1963.

10. The Angiosperm Phylogeny Group: An update of the Angiosperm Phylogeny Group classification for the orders and families of flowering plants: APG III. >Bot J Linn Soc 2009, 161:105-121.

11. Anderson $C L$, Bremer $K$, Friis EM: Dating phylogenetically basal eudicots using $r b c L$ sequences and multiple fossil reference points. Am J Bot 2005, 92(10):1737-1748.

12. Sanderson MJ, Doyle JA: Sources of error and confidence intervals in estimating the age of angiosperms from $r b c L$ and $18 \mathrm{~S}$ rDNA data. Am J Bot 2001, 88(8):1499-1516.

13. Barker NP, Weston PH, Rutschmann F, Sauquet H: Molecular dating of the 'Gondwanan' plant family Proteaceae is only partially congruent with the timing of the break-up of Gondwana. J Biogeogr 2007, 34:2012-2027.

14. Dettman ME, Jarzen DM: The early history of the Proteaceae in Australia: the pollen record. Aust Syst Bot 1998, 11:401-438.

15. Sauquet $H$, Weston PH, Anderson CL, Barker NP, Cantrill DJ, Mast AR, Savoainen V: Contrasted patterns of hyperdiversification in Mediterranean hotspots. Proc Natl Acad Sci USA 2009, 106(1):221-225.

16. Valente LM, Reeves G, Schnitzler J, Mason IP, Fay MF, Rebelo TG, Chase MW, Barraclough TG: Diversification of the African genus Protea (Proteaceae) in the Cape biodiversity hotspot and beyond: equal rates in different biomes. Evolution 2010, 64:745-760.

17. Mast $\mathrm{AR}$, Willis $\mathrm{CL}$, Jones $\mathrm{EH}$, Downs KM, Weston PH: A smaller Macadamia from a more vigile tribe: Inference of phylogenetic relationships, divergence times and diaspore evolution in Macadamia and relatives (Tribe Macadamieae; Proteaceae). Am J Bot 2008, 95(7):843-870.

18. Duchenne $D$, Bromham $L$ : Rates of molecular evolution and diversification in plants: chloroplast substitution rates correlate with species-richness in the Proteaceae. BMC Evol Biol 2013, 13:65.

19. Moore M, Dhingra A, Soltis PS, Shaw R, Farmarie WG, Folta KM, DE Soltis: Rapid and accurate pyrosequencing of angiosperm plastid genomes. BMC Plant Biol 2006, 6:17.

20. Shinozaki K, Ohme M, Tanaka M, Wakasugi T, Hayashida N, Matsubayashi T, Zaita N, Chunwongse J, Obokata J, Yamaguchi-Shinozaki K, Ohto C, Torazawa K, Meng BY, Sugita M, Deno H, Kamogashira T, Yamada K, Kusuda J, Takaiwa F, Kato A, Tohdoh N, Shimada H, Sugiura M: The complete nucleotide sequence of tobacco chloroplast genome: its gene organization and expression. EMBO J 1986, 5:2043-2049.

21. Wicke S, Schneeweiss GM, dePamphilis CW, Muller KF, Quandt D: The evolution of the plastid chromosome in land plants: Gene content, gene order, gene function. Plant Mol Biol 2011, 76:273-297.

22. Delannoy E, Fujii S, Colas des Francs-Small C, Brundrett M, Small I: Rampant gene loss in the underground orchid Rhizanthella gardneri highlights evolutionary constraints on plastid genomes. Mol Biol Evol 2011, 28(7):2077-2086

23. Molina J, Hazzouri KM, Nickrent D, Geisler M, Meyer RS, Pentony MM, Flowers JM, Pelser P, Barcelona J, Inovejas SA, Uy I, Yuan W, Wilkins O, Claire-Iphanise M, LockLear S, Concepcion GP, Purugganan MD: Possible loss of the chloroplast genome in the parasitic flowering plant Rafflesia lagascae (Rafflesiaceae). Mol Biol Evol 2014, 31(4):793-803.

24. Zhang $\mathrm{H}$, Li C, Miao H, Xiong S: Insights from the complete chloroplast genome into the evolution of Sesamum indicum L. PLOS ONE 2013, 8(11): e80508.

25. Nugent JM, Herbon AL: Unusual structure of geranium chloroplast DNA: A triple-sized inverted repeat, extensive gene duplications, multiple inversions, and two repeat families. Proc Natl Acad Sci USA 1987, 84:769-773

26. Martínez-Alberola F, del Campo EM, Lázaro-Gimeno D, MezquitaClaramonte S, Molins A, Mateu-Andrés I, Pedrola-Monfort J, Casano LM, Barreno E: Balanced gene losses, duplications and intensive rearrangements led to an unusual regularly sized genome in Arbutus unedo chloroplasts. PLOS ONE 2013, 8(11):e79685.

27. Sun Y, Moore MJ, Meng A, Soltis PS, Soltis DE, Li J, Wang H: Complete plastid genome sequencing of Trochodendraceae reveals a significant expansion of the inverted repeat and suggests a Paleogene divergence between the two extant species. PLOS ONE 2013, 8(4):e60429.

28. Shi C, Liu Y, Huang H, Xia E, Zhang H, Gao L: Contradiction between plastid gene transcription and function due to complex posttranscriptional splicing: an exemplary study of ycf15 function and evolution in angiosperms. PLOS ONE 2013, 8(3):e59620.
29. The Angiosperm Phylogeny Group: An ordinal classification for the families of flowering plants. Ann Mo Bot Gar 1998, 85(4):531-553.

30. Soltis DE, Soltis PS, Chase MW, Mort ME, Albach DC, Zanis M, Savolainen V, Hahn WH, Hoot SB, Fay MF, Axtell M, Swensen SM, Prince LM, Kress WJ, Nixon KC, Farris JS: Angiosperm phylogeny inferred from 18S rDNA, rbcL, and atpB sequences. Bot J Linn Soc 2000, 133:381-461.

31. Weston PH: What has molecular systematics contributed to our knowledge of the plant family Proteaceae? Methods Mol Biol 2014, 1115:365-397.

32. Barniske A, Borsch T, Müller K, Krug M, Worberg A, Neinhuis C, Quandt D: Phylogenetics of early branching eudicots: comparing phylogenetic signal across plastid introns, spacers, and genes. J Syst Evol 2012, 50(2):85-108.

33. Parks $M$, Cronn $R$, Liston $A$ : Increasing phylogenetic resolution at low taxonomic levels using massively parallel sequencing of chloroplast genomes. BMC Biol 2009, 7:84.

34. Nock CJ, Waters DL, Edwards MA, Bowen SG, Rice N, Cordeiro GM, Henry RJ: Chloroplast genome sequences from total DNA for plant identification. Plant Biotechnol J 2011, 9(3):328-333.

35. Hollingsworth PM, Graham SW, Little DP: Choosing and using a plant DNA barcode. PLOS ONE 2011, 6(5):e19254

36. Arthofer W, Schüler S, Steiner FM, Schlick-Steiner BC: Chloroplast DNAbased studies in molecular ecology may be compromised by nuclearencoded plastid sequence. Mol Ecol 2010, 19:3853-3856.

37. Smith DR, Crosby K, Lee RW: Correlation between nuclear plastid abundance and plastid number supports the limited transfer window hypothesis. Genome Biol Evol 2011, 3:365-371.

38. Ferrarini M, Moretto M, Ward JA, Surbanovski N, Stevanovi V, Giongo L, Viola R, Cavalieri D, Velasco R, Cestaro A, Sargent DJ: An evaluation of the PacBio RS platform for sequencing and de novo assembly of a chloroplast genome. BMC Genomics 2013, 14(1):670.

39. Stull GW, Moore MJ, Mandala VS, Douglas NA, Kates HR, Qi X, Brockington SF, Soltis PS, Soltis DE, Gitzendanner MA: A targeted enrichment strategy for massively parallel sequencing of angiosperm plastid genomes. Appl Plant Sci 2013, 1(2):1200497.

40. McPherson $\mathrm{H}$, van der Merwe M, Delaney SK, Edwards MA, Henry RJ, Mclntosh E, Rymer RD, Milner ML, Siow J, Rossetto M: Capturing chloroplast variation for molecular ecology studies: a simple next generation sequencing approach applied to a rainforest tree. BMC Ecol 2013, 13:8

41. Provan J, Powell W, Hollingsworth PM: Chloroplast microsatellites: new tools for studies in plant ecology and evolution. Trends Ecol Evol 2001, 16(3):142-147.

42. Neville PG, Bradbury D, Williams A, Tomlinson S, Krauss SL: Genetic and palaeo-climatic evidence for widespread persistence of the coastal tree species Eucalyptus gomphocephala (Myrtaceae) during the Last Glacial Maximum. Ann Bot 2014, 113(1):55-67.

43. Zhang Z, Schwartz S, Wagner L, and Miller W: A greedy algorithm for aligning DNA sequences. J Comput Biol 2000, 7(1-2):203-14.

44. Kurtz S, Phillippy A, Delcher AL, Smoot M, Shumway M, Antonescu C, Salzberg SL: Versatile and open software for comparing large genomes. Genome Biology 2004, 5:R12.

45. Li H, Durbin R: Fast and accurate short read alignment with BurrowsWheeler Transform. Bioinformatics 2009, 25:1754-60.

46. Bonfield JK, Whitwham A: Gap5-editing the billion fragment sequence assembly. Bioinformatics 2010, 26(14):1699-1703.

47. Wyman SR, Jansen RK, Boore JL: Automatic annotation of organellar genomes with DOGMA. Bioinformatics 2004, 20:3252-3255.

48. Katoh K, Misawa K, Kuma K, Miyata T: MAFFT: a novel method for rapid multiple sequence alignment based on fast Fourier transform. Nuc Acids Res 2002, 30:3059-3066.

49. Frazer KA, Pachter L, Poliakov A, Rubin EM, Dubchak I: VISTA: computational tools for comparative genomics. Nuc Acids Res 2004, 32(Suppl2):W273-W279.

50. Faircloth B: MSATCOMMANDER: detection of microsatellite repeat arrays and automated, locus-specific primer design. Mol Ecol Res 2008, 8:92-94.

51. Lanfear R, Calcott B, Ho SYW, Guindon S: PartitionFinder: combined selection of partitioning schemes and substitution models for phylogenetic analyses. Mol Biol Evol 2012, 29(6):1695-1701.

52. Stamatakis A: Raxml-vi-hpc: maximum likelihood-based phylogenetic analyses with thousands of taxa and mixed models. Bioinformatics 2006, 22:2688-2690. 
doi:10.1186/1471-2164-15-S9-S13

Cite this article as: Nock et al:: Complete chloroplast genome of

Macadamia integrifolia confirms the position of the Gondwanan early-

diverging eudicot family Proteaceae. BMC Genomics 2014 15(Suppl 9):S13.

Submit your next manuscript to BioMed Central and take full advantage of:

- Convenient online submission

- Thorough peer review

- No space constraints or color figure charges

- Immediate publication on acceptance

- Inclusion in PubMed, CAS, Scopus and Google Scholar

- Research which is freely available for redistribution

Submit your manuscript at 